\title{
FACTORIZATION OF DIFFERENTIAL IDEALS
}

\author{
HENRY J. RIBLET
}

1. Introduction. With the ultimate objective of introducing number theoretical considerations into the theory of algebraic differential fields, ${ }^{1}$ conditions on a differential ring $\Re$ are given which are sufficient to insure that any differential ideal in it may be uniquely expressed as the product of a finite number of prime differential ideals. This program is justified by its intrinsic interest and the fact that the existence of such conditions suggests the possibility of obtaining a number theoretical complement for the theory of differential ideals in the ring of differential forms as developed by Ritt, Raudenbush and others, ${ }^{2}$ paralleling the interdependent, classical theories of polynomial and number ideals.

With an additional axiom and certain alterations of the definitions, the proof of the basic theorems follows the pattern used by van der Waerden ${ }^{3}$ in presenting the method of Krull. ${ }^{4}$ Our procedure will be to discuss in detail only those questions raised by the altering of definitions.

2. Definitions. By a differential ring $\Re$, we shall mean a commutative ring with a unit element but no divisors of zero which is closed under differentiation. Its quotient field will be denoted by $\Omega$. A differential ideal $\mathfrak{a}$ in $\Re$ is an ordinary ideal closed with respect to differentiation. $\Re$ will satisfy the ascending chain condition for differential ideals if every sequence of differential ideals, each properly containing the previous one, is finite in length. A differential ideal is said to be divisorless if the only differential ideal which properly contains it is the unit ideal $\Re$. A principal differential ideal is a differential ideal which is principal in the usual sense. The unit ideal is an example. $\Re$ will be said to be integrally closed if each element in $\Omega$, having the property that all its integral powers have a fixed denominator, is in $\Re$. A differential ideal $\mathfrak{p}$ in $\Re$ will be called

Presented to the Society, April 26, 1940 under the title Factorization of differential ideals in an algebraic field; received by the editors November 6, 1941.

${ }^{1} \mathrm{H}$. J. Riblet, Algebraic differential fields, American Journal of Mathematics, vol. 53 (1941), pp. 339-446.

${ }^{2} \mathrm{~J} . \mathrm{F}$. Ritt, Algebraic aspects of the theory of differential equations, American Mathematical Society Semicentennial Publications, vol. 2, bibliography.

${ }^{3}$ B. L. van der Waerden, Moderne Algebra, vol. 2, p. 85.

${ }^{4} \mathrm{~W}$. Krull, Zur Theorie der allgemeinen Zahlringe, Mathematische Annalen, vol. 99 (1928), pp. 51-70. 
prime if the existence of two differential ideals $\mathfrak{a}$ and $\mathfrak{b}$ such that $\mathfrak{a} \cdot \mathfrak{b} \equiv 0(\mathfrak{p})$ implies that $\mathfrak{a} \equiv 0(\mathfrak{p})$ or $\mathfrak{b} \equiv 0(\mathfrak{p})$.

It may be commented that the definition of an integrally closed differential ring is taken from Krull. ${ }^{5}$ The more familiar definition ${ }^{6}$ is a consequence of this plus the stronger assumption that an ascending chain condition holds for all ideals in $\Re$. It should be observed also that this definition of primeness for differential ideals is not equivalent to that of Raudenbush. ${ }^{7}$ If we restrict ourselves to the perfect differential ideals ${ }^{8}$ of $\Re$, however, the two definitions coincide.

3. Axioms. Let $\Re$ be a differential ring in which the following axioms are satisfied:

I. The ascending chain condition for differential ideals in $\Re$ holds.

II. Every prime differential ideal in $\Re$ is divisorless.

III. Every prime differential ideal in $\Re$ contains a principal differential ideal.

IV. $\Re$ is integrally closed.

With these axioms, we then prove the two basic theorems on prime factorization:

THEOREM 1. Every differential ideal in $\Re$ is a product of a finite number of prime differential ideals.

THEOREM 2. If $\mathfrak{a} \equiv 0(\mathfrak{b})$, then each prime factor of $\mathfrak{a}$ always occurs at least as often as a prime factor of $\mathfrak{b}$.

The proofs are identical with those given in Van der Waerden.9 The only question occurs in using the symbol $\mathfrak{p}^{-1}$ to denote the set of elements in $\Re$ such that if $a$ is one of them, $a \cdot \mathfrak{p}$ is in $\Re$. It is clear that $\mathfrak{p}^{-1}$ is closed with respect to addition and with respect to multiplication by elements of $\Re$. It is also true that it is closed with respect to differentiation for, if $a \in p^{-1}$ and $p \in \mathfrak{p}$, then $a \cdot p \in \Re$. Hence $a \cdot p^{(1)}-a^{(1)} \cdot p \in \Re$, where (1) denotes differentiation. Now $a \cdot p^{(1)} \in \Re$, so $a^{(1)} \cdot p \in \Re$; but $p$ is any member of $p$ and accordingly $a^{(1)} \in \mathfrak{p}^{-1}$. Note that Axioms III and IV allow us to prove that $\mathfrak{p} \cdot \mathfrak{p}^{-1}=\Re$.

From the two theorems, it follows that divisibility implies factorization and that each differential ideal in $\Re$ may be expressed

${ }^{5}$ W. Krull, ibid., p. 60.

6 B. L. van der Waerden, loc. cit., p. 90.

${ }^{7} \mathrm{H}$. W. Raudenbush, Jr., Ideal theory and algebraic differential equations, Transactions of this Society, vol. 36 (1934), p. 365.

${ }^{8}$ H. W. Raudenbush, Jr., ibid., p. 362.

${ }^{9}$ B. L. van der Waerden, loc. cit., pp. 97-100. 
uniquely as a product of a finite number of prime differential ideals. The consistency of the axioms is easily shown. If we define differentiation in the ring $C(x)$, obtained by adjoining $x$ to the field of the rational numbers, in any way so as to leave it closed, it may be shown that Axioms I-IV are always satisfied. In $C(x, y)$ differentiation may be defined in such way that the statement as above still holds. This is of interest because every ordinary ideal in $C(x, y)$ may not be expressed uniquely as a product of a finite number of prime ideals.

Hofstra College

\section{ON THE ITERATION OF LINEAR HOMOGENEOUS TRANSFORMATIONS}

\section{ARNOLD DRESDEN}

1. Statement of problem. The question which this note tries to answer is that of determining under what conditions on the matrix $\left(a_{i j}\right)$, $(i, j=1, \cdots, n)$, the $n$-fold multiple sequence of complex numbers $x_{k}, x_{k}^{\prime}, \cdots, x_{k}^{(m)}, \cdots(k=1,2, \cdots, n)$ obtained by iteration of the linear homogeneous transformation $x_{k}^{\prime}=a_{k j} x_{j}$ will converge for every initial set $x=\left(x_{1}, x_{2}, \cdots, x_{n}\right)$. Convergence is to be understood in the sense that there exists a set $X_{1}, X_{2}, \cdots, X_{n}$ such that, for $k=1,2, \cdots, n, x_{k}^{(m)} \rightarrow X_{k}$, as $m \rightarrow \infty$.

2. Jordan normal form. We begin by recalling that a matrix $A=\left(a_{i j}\right)$ with complex elements is similar to its Jordan normal form $J_{0}$. This means that there exists a unimodular matrix $P$, such that $A=P^{-1} J_{0} P$ and $J_{0}=P A P^{-1}$, where $J_{0}$ is the direct sum of Jordan matrices $J_{1}, \cdots, J_{N}$. To each elementary divisor $\left(\lambda-\lambda_{\rho}\right)^{e_{\rho}}$ of the characteristic matrix $\lambda I-A(\rho=1,2, \cdots, N)$ and $e_{1}+e_{2}+\cdots+e_{N}$ $=n$, corresponds a Jordan matrix $J_{\rho}$. If $e_{\rho}>1$, then $J_{\rho}$ has zero elements everywhere, except in the principal diagonal, all of whose elements are $\lambda_{\rho}$, and in the diagonal immediately below the principal diagonal, all of whose elements are $1 .{ }^{1}$ If $e_{\rho}=1$, then $J_{\rho}$ consists of the single element $\lambda_{\rho}$.

It follows that any integral power of $J_{0}$ is the direct sum of the same powers of the Jordan matrices $J_{\rho}$. Let us now denote by $J$ an arbitrary Jordan matrix of order $n>1$,

Received by the editors August 25, 1941.

${ }^{1}$ See, for example, MacDuffee, Introduction to Abstract Algebra, p. 241. 